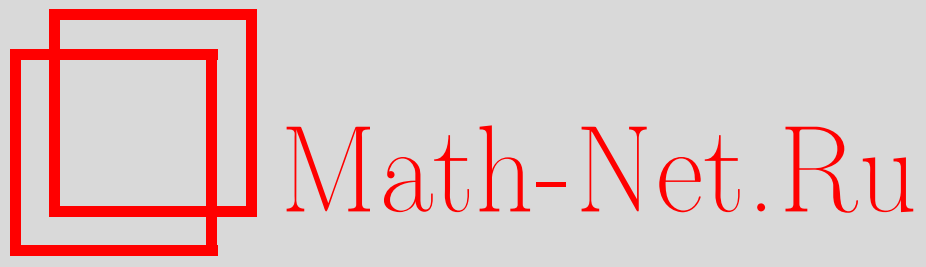

А. Н. Колмогоров, Отзыв о работах И. М. Гельфанда и М.А. Наймарка по унитарным представлениям групп Ли, Функи. анализ и его прил., 2003, том 37, выпуск 4, 8

DOI: https://doi.org/10.4213/faa3009

Использование Общероссийского математического портала MathNet.Ru подразумевает, что вы прочитали и согласны с пользовательским соглашением

http://www. mathnet.ru/rus/agreement

Параметры загрузки:

IP: 52.87 .193 .239

26 апреля 2023 г., 17:50:56

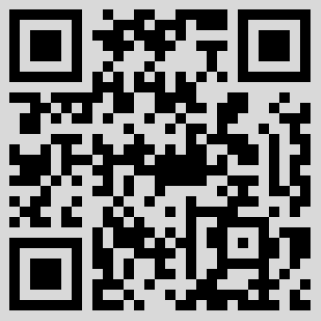




\section{Отзыв о работах И. М. Гельфанда и М. А. Наймарка по унитарным представлениям групп Ли}

Наиболее глубокие конкретные математические вопросы связаны с линейными представлениями групп Ли. В коммутативном и компактном случае все такие представления разлагаются на неприводимые конечномерные, т. е. осуществимые при помощи матриц. Но уже имеющая фундаментальное значение для физики группа Лоренца, группа движений плоскости Лобачевского и ряд других классических групп имеют только очень бедный запас конечномерных представлений, из которых, например, нельзя получить «регулярное» представление функциями с интегрируемым квадратом, заданными на самой группе.

Поэтому возникает задача найти для основных классических групп Ли все их бесконечномерные неприводимые унитарные представления (конечномерные уже были известны) и исследовать возможность разложения любых унитарных представлений на неприводимые. Эта задача и была разрешена после преодоления больших трудностей И. М. Гельфандом и М. А. Наймарком. Их работы имеют вполне классический характер по методам, конкретности результатов, данных в виде явных формул, и широте полученных и ожидаемых применений.

Особый интерес для современной физики имеет нахождение всех неприводимых унитарных представлений группы Лоренца и получение из них всех унитарных представлений этой группы. Эта задача решена в работе [1]. О ее значении для физики см. [3]. Важность вопроса была ясна и зарубежным ученым. Но Дирак в 1945 году смог только указать несколько случайных бесконечномерных представлений группы Лоренца, которые все оказались разложимыми. Только после работ И. М. Гельфанда и $M$. А. Наймарка возникла обширная литература за рубежом.

С математической стороны еще большие трудности преодолены в фундаментальной монографии [3], вышедшей в 1950 году. Построенная в ней теория обобщенных «сферических функций» и многие другие результаты представляются нам весьма существенными для анализа.

В целом эти исследования представляют собой одно из самых замечательных явлений в современной математике вообще.

1. Гельфанд И. М., Наймарк М. А. Унитарные представления группы Лоренца. Изв. АН СССР, сер. матем., 11, 411-504 (1947).

2. Гельфанд И. М., Яглом А. М. Общие инвариантные уравнения и бесконечномерные представления группы Лоренца. ЖЭТФ, 18, 703-733 (1948).

3. Гельфанд И. М., Наймарк М. А. Унитарные представления классических групп. Труды МИАН им. В. А. Стеклова, 36, 1-288 (1950).

\section{Отзыв о работах И. М. Гельфанда}

\section{(в связи с его выдвижением в действительные члены АН СССР)}

Основные научные достижения Израиля Моисеевича Гельфанда относятся к функциональному анализу, а в той или иной мере связаны с использованием методов и идей функционального анализа почти все его работы. После того 